\title{
Enzymatic hydrolysis of PTT polymers and oligomers
}

\author{
A. Eberl ${ }^{\mathrm{a}, \mathrm{b}}$, S. Heumann ${ }^{\mathrm{a}, \mathrm{b}}$, R. Kotek ${ }^{\mathrm{c}}$, F. Kaufmann ${ }^{\mathrm{d}}$, S. Mitsche ${ }^{\mathrm{e}}$, \\ A. Cavaco-Paulo ${ }^{f}$, G.M. Gübitz ${ }^{\mathrm{b}, *}$ \\ ${ }^{a}$ Research Centre Applied Biocatalysis, Petersgasse 14, A-8010 Graz, Austria \\ ${ }^{\mathrm{b}}$ Graz University of Technology, Department of Environmental Biotechnology, Petersgasse 12, A-8010 Graz, Austria \\ ${ }^{\mathrm{c}}$ College of Textiles, Textile Engineering, Chemistry and Science Department, North Carolina State University, USA \\ d CIBA Inc., Basel, Switzerland \\ e Graz University of Technology, Research Institute for Electron Microscopy, Steyrergasse 17, A-8010 Graz, Austria \\ ${ }^{\mathrm{f}}$ University of Minho, Department of Textile Engineering, 4800 Guimaraes, Portugal
}

\section{A R T I C L E I N F O}

\section{Article history:}

Received 8 August 2007

Received in revised form 4 February 2008

Accepted 18 February 2008

\section{Keywords:}

Poly(trimethylene terephthalate)

Polyester

Polyesterase

Cutinase

\begin{abstract}
A B S T R A C T
Oligomers and polymers (film, fabrics) of the linear aromatic polyester poly(trimethylene terephthalate) (PTT) were treated with polyesterases from Thermomyces lanuginosus, Penicillium citrinum, Thermobifida fusca and Fusarium solani pisi. The cutinase from T. fusca was found to release the highest amounts of hydrolysis products from PTT materials and was able to open and hydrolyse a cyclic PTT dimer according to RP-HPLC-UV detection. In contrast, the lipase from T. lanuginosus also showed activity on the PTT fibres and on bis(3-hydroxypropyl) terephthalate (BHPT) but was not able to hydrolyse the polymer film, mono(3-hydroxypropyl) terephthalate (MHPT) nor the cyclic dimer of PTT. As control enzymes inhibited with mercury chloride were used. Surface hydrophilicity changes were investigated with contact angle measurements and the degree of crystallinity changes were determined with DSC.
\end{abstract}

(C) 2008 Elsevier B.V. All rights reserved.

\section{Introduction}

Poly(trimethylene terephthalate) (PTT) was first synthesized in 1941 but due to the high costs of high-quality propanediol, one of the raw materials to produce PTT, it was not commercially available. In 1998 PTT was finally introduced into the market by Shell Chemicals under the trade name Corterra since an economical process for the production of 1,3-propanediol had been developed. It belongs to the group of linear aromatic polyesters next to poly(ethylene terephthalate) and poly(butylene terephthalate) with three methylene groups in the glycol repeating unit. The odd number of methylene units affects the physical and chemical structure of PTT resulting in several excellent properties as the high elastic recovery and dyeing ability (Houck et al., 2001). Initially PTT was intended for the carpeting market but due to its processability like spinning and dyeing properties it resulted to be suitable for the fibre market in the fields of sportswear and active wear as well (Houck et al., 2001; Wu et al., 2005). DuPont ${ }^{\mathrm{TM}}$ introduced the fibre under the trade name Sorona ${ }^{\circledR}$ and the polymer is additionally used in many other end use applications for films, filaments and engineering plastics. Next to the source of petrochemical-based materials DuPont ${ }^{\mathrm{TM}}$ developed a

\footnotetext{
* Corresponding author.

E-mail address: guebitz@tugraz.at (G.M. Gübitz).
}

new process that uses renewable sources to manufacture 1,3 propanediol.

Besides the excellent properties of PTT for several applications, a higher hydrophilicity of the particularly hydrophobic polymer is essential. To increase hydrophilicity methods like alkaline treatment and plasma treatment are usually used. A rather new interesting alternative is the use of enzymes in surface modification of synthetic fibres (Silva et al., 2005; Fischer-Colbrie et al., 2004; Araujo et al., 2007; Heumann et al., 2006; Nimchua et al., 2007).

It was not until the past few years that there is evidence in microbial attack on aromatic polyesters (Mueller, 2006; Mueller et al., 2005; Vertommen et al., 2005). Before aromatic polyesters were regarded to be non-biodegradable (Mueller et al., 2001). Research was carried out in synthetic aliphatic-aromatic polyesters providing good mechanical properties resulting from of the aromatic polyesters and show acceptable biodegradability, since microbiological attack of many aliphatic polyesters has been known for many years (Marten et al., 2003, 2005). An important part in polymer hydrolysis next to the degree of crystallinity is the mobility of the polymer chains. This correlates with the difference of the melting temperature of the crystalline fraction of the polyester and the temperature at which degradation takes place (Marten et al., 2005).

A few years ago the thermophilic actinomycete Thermobifida fusca was isolated and the polyester degrading enzyme was characterized to be a hydrolase combining the properties of a lipase and esterase (Gouda et al., 2002; Kleeberg et al., 2005). These 
enzymes are often named as cutinases and therefore used in the further study. The recombinant enzyme from $T$. fusca is used in this study (Dresler et al., 2006) next to a triacylglycerol lipase from Thermomyces lanuginosus.

This paper is the first scientific report focusing on enzymatic hydrolysis of the promising aromatic polyester poly(trimethylene terephthalate) using diverse enzymes belonging to the "class of polyesterases".

\section{Materials and methods}

\subsection{Chemicals and enzymes}

\subsubsection{Chemicals}

Methanol and acetonitrile used were HPLC grade quality and purchased from Roth (Carl Roth GmbH, Karlsruhe, Germany) and VWR Prolabo, respectively. Diethylether p.a. was purchased by Roth and deionised water was used. Astrazone Blue BG (C.I. Basic Blue 3) was a kind gift of Dystar Textilfarben (Frankfurt, Germany). All other chemicals were analytical grade from SIGMA.

\subsubsection{Enzymes}

The cutinases from T. fusca and Fusarium solani pisi were produced as previously described (Mueller et al., 2005; Araujo et al., 2007). The cutinase from T. fusca was stored as lyophilised powder with a protein content of $5 \%$ while the $F$. solani pisi was stored as buffered solution at $4{ }^{\circ} \mathrm{C}$ as resulting from purification. The lipase from T. lanuginosus is available from Novozymes and the Penicillium citrinum enzyme was produced and purified as previously reported (Liebminger et al., 2007).

\subsection{Substrates}

\subsubsection{Polymeric substrates}

For the production of poly(trimethylene terephthalate) (PTT) films PTT granules were dried under vacuum at $120^{\circ} \mathrm{C}$. Dried PTT granules $(1 \mathrm{~g})$ were pressed at $248-250^{\circ} \mathrm{C}$ between Teflon sheets of $1 \mathrm{~mm}$ thickness under $350 \times 10^{5}$ Pa pressure. After 6 min of preheating the granules were pressed for $30 \mathrm{~min}$ followed by cooling down in the water bath at $25 \pm 1^{\circ} \mathrm{C}$ for $30 \mathrm{~min}$.

Beside films two un-dyed and dyed knit PTT fabrics were provided by Shell Chemicals. The fabric was dyed with a disperse blue dyestuff at $95^{\circ} \mathrm{C}$. The cyclic dimer of PTT, which is an unwanted by-product during PTT production was obtained from a PTT manufacturing plant (DuPont, Kinston, NC) and was further purified (Pang et al., 2006).

The degrees of crystallinity of the polymer PTT substrates were measured with Differential Scanning Calorimetry (DSC) runs and determined to be $21.1 \%$ for PTT-films, $45.4 \%$ for the dyed PTT fabric and $36.9 \%$ for the undyed PTT fabric.

Crystallinity analysis for all polymeric substrates was carried out using a PerkinElmer PYRIS DIAMOND DSC. About $4 \mathrm{mg}$ of the sample were taken from the test substance and weighed accurately to $1 \mu \mathrm{g}$. Heating was carried out in a nitrogen atmosphere starting from room temperature to $300^{\circ} \mathrm{C}$ in steps of $10^{\circ} \mathrm{C} \mathrm{min}^{-1}$ in standard aluminium pans. The heats of fusion, $\Delta H_{\mathrm{m}}$, and cold crystallization, $\Delta H_{\mathrm{c}}$, were determined by integrating the areas $\left(\mathrm{Jg}^{-1}\right)$ under the peaks. The percent crystallinity was determined using the following equation (Sichina, 2000):

crystallinity $(\%)=\frac{\left|\Delta H_{\mathrm{m}}\right|-\left|\Delta H_{\mathrm{c}}\right|}{\Delta H_{\mathrm{m}}^{\circ}} \times 100 \%$

$\Delta H_{\mathrm{m}}$ is the heat of fusion $\left(\mathrm{Jg}^{-1}\right) ; \Delta H_{\mathrm{c}}$ is the heat of crystallization $\left(\mathrm{Jg}^{-1}\right) ; \Delta H_{\mathrm{m}}{ }^{\circ}$ is the heat of fusion for a $100 \%$ crystalline polymer $\left(\mathrm{Jg}^{-1}\right) ; \Delta H_{\mathrm{m}}{ }^{\circ}$ is a reference value representing the heat of melting in a $100 \%$ crystalline polymer and was estimated to be $145 \mathrm{Jg}^{-1}$ (Chen et al., 2007).

\subsubsection{Synthesis of bis(3-hydroxypropyl) terephthalate (BHPT)}

Bis(3-hydroxypropyl) terephthalate (BHPT) which is a bis-ester of terephthalic acid, was synthesized according to a method modified by Lyoo et al. (2000). The synthesis was carried out via transesterification reaction of dimethyl terephthalate (DMT) with 1,3-propanediol (1,3-PD) using titanium tetrabutoxide (TBT) as a catalyst. The transesterification of DMT (6.68 g: $0.04 \mathrm{~mol})$ with 1,3-propanediol $(60 \mathrm{ml}: 0.8 \mathrm{~mol})$ in the presence of TBT $(4 \mu \mathrm{l}$ : $1 \times 10^{-5} \mathrm{~mol}$ ) was performed in a round bottom flask equipped with a water separator and a reflux condenser. The mixture of DMT, 1,3-PD and catalyst was heated at $160^{\circ} \mathrm{C}$ for $5 \mathrm{~h}$. After stopping the reaction, the product was washed with water and rewashed with ethyl ether to remove residual 1,3-PD. The crude product was crystallized from water. The melting point was $77-79^{\circ} \mathrm{C}$.

\subsection{Monitoring of hydrolysis products of PTT substrates}

\subsubsection{Sample preparation}

Three pieces of $5 \mathrm{mg}$ PTT film $(4 \mathrm{~mm} \times 5 \mathrm{~mm})$ were treated in Eppendorf tubes with $0.5 \mathrm{ml}$ enzyme solution. The protein content of enzyme solutions was measured according to the method of Lowry et al. (1951). Cutinase from T. fusca was used in a concentration of $0.2 \mathrm{~g}_{\text {protein }} \mathrm{l}^{-1}$ in $50 \mathrm{mM}$ phosphate buffer, $\mathrm{pH} 7$ $\left(\mathrm{Na}_{2} \mathrm{HPO}_{4} / \mathrm{KH}_{2} \mathrm{PO}_{4}\right)$. Enzymes from $F$. solani and $P$. citrinum were used in concentrations of $1.5 \mathrm{~g}$ protein $\mathrm{I}^{-1}$ and lipase from $T$. lanuginosus of $38 \mathrm{~g}$ protein $\mathrm{l}^{-1}$. Incubation with cutinase from $T$. fusca was carried out at $60^{\circ} \mathrm{C}$ at $450 \mathrm{rpm}$ (thermomixer comfort, Eppendorf); the temperature for the incubations with enzyme from $P$. citrinum and $F$. solani and the lipase from T. lanuginosus was set to $37^{\circ} \mathrm{C}$. After the intended incubation times the samples were put on ice to stop the reaction. For protein precipitation the samples for HPLC measurement were treated $1: 1(\mathrm{v} / \mathrm{v})$ with methanol abs. on ice. After $15 \mathrm{~min}$ the samples were centrifuged at $16,000 \times \mathrm{g}$ for $15 \mathrm{~min}$ at $0{ }^{\circ} \mathrm{C}$ (HERMLE Z $300 \mathrm{~K}$, Wehingen, Germany). Supernatant for measurement was directly brought to a HPLC vial and acidified by adding $1 \mu \mathrm{l}$ of $\mathrm{HCl}$ conc.

Similarly, incubations for the two different PTT fabrics were carried out. In the case of the cyclic dimer $10 \mathrm{mg}$ were weighed into Eppendorf tubes and incubated with $0.5 \mathrm{ml}$ enzyme solution. Further sample preparation was carried out as described above. Alkaline treatment for white PTT fabrics was carried out with $5 \mathrm{M}$ $\mathrm{NaOH}$ at $95^{\circ} \mathrm{C}$ for $30 \mathrm{~min}$.

For contact angle measurements of PTT films pieces of $4 \mathrm{~cm} \times 4 \mathrm{~cm}$ were incubated in $15 \mathrm{ml}$ of enzyme solution (protein concentrations see above) for $120 \mathrm{~h}$. After incubation, the films were washed twice with phosphate buffer for $30 \mathrm{~min}$ at $60^{\circ} \mathrm{C}$ and additionally twice with deionised water at the same conditions.

To study the enzymatic hydrolysis of BHPT, $2.97 \mathrm{mg}$ BHPT were dissolved in $50 \mathrm{mM}$ phosphate buffer to give a $0.105 \mathrm{mM}$ solution. To $500 \mu \mathrm{l}$ of BHPT $500 \mu \mathrm{l}$ enzyme solution were added for the different incubation times. Protein precipitation was achieved as described above.

Blank incubations were also carried out with the substrates in the same buffer without enzyme addition as well as with the enzyme solutions only. Additionally, inhibition experiments for the PTT materials with $\mathrm{HgCl}_{2}$ were carried out with a final concentration of $1 \mathrm{mM}$ of $\mathrm{HgCl}_{2}$ in the samples.

Weight loss of PTT was additionally calculated for alkaline treated as well as enzyme treated white PTT fabric. Fabrics were equilibrated over night in a desiccator and weighed on an analytical balance. Therefore after incubation the fabric pieces were washed 
and dried overnight at $50^{\circ} \mathrm{C}$ in the drying oven, cooled down to ambient temperature in a desiccator and then weighed again.

\subsubsection{HPLC-UV-detection}

The HPLC equipment used was a DIONEX P-580 PUMP (Dionex Cooperation, Sunnyvale, USA), with an ASI-100 automated sample injector and a PDA-100 photodiode array detector.

For analysis of terephthalic acid (TA), mono(3-hydroxypropyl) terephthalate (MHPT) and bis(3-hydroxypropyl) terephthalate (BHPT) a reversed phase column RP-C18 (Discovery ${ }^{\circledR} \mathrm{HS}-\mathrm{C} 18,5 \mu \mathrm{m}$, $150 \mathrm{~mm} \times 4.6 \mathrm{~mm}$ with precolumn, Supelco, Bellefonte, USA) was used. Analysis was carried out with 25\% acetonitrile, $20 \% 10 \mathrm{mM}$ sulphuric acid and $55 \%(\mathrm{v} / \mathrm{v})$ water as eluent. The flow rate was set to $1 \mathrm{ml} \mathrm{min}^{-1}$ and the column was maintained at a temperature of $40^{\circ} \mathrm{C}$. The injection volume was $10 \mu \mathrm{l}$. Detection was performed with a photodiode array detector at the wavelength of $241 \mathrm{~nm}$.

\subsubsection{NMR and MS spectra}

NMR spectra were recorded on a Varian Unity Inova 500 instrument; ${ }^{13} \mathrm{C}$ data were measured. The MS spectra were acquired with an Agilent Ion Trap SL with electrospray ionisation with direct infusion (flow $=5 \mu \mathrm{min}^{-1}$ for BHPT) as well as coupled to the Dionex HPLC-UVD-system (flow $=1 \mathrm{ml} \mathrm{min}^{-1}$ for MHPT). BHPT was measured in positive ion mode and MHPT in negative ion mode and the electrospray voltage was set to $-3500 \mathrm{~V}$ and $+3500 \mathrm{~V}$, respectively. Dry gas $\left(51 \mathrm{~min}^{-1}\right)$ temperature was set to $325^{\circ} \mathrm{C}$, nebulizer to 15 psi for BHPT. For MHPT the dry gas flow was set to $12 \mathrm{l} \mathrm{min}^{-1}$ with a temperature of $350^{\circ} \mathrm{C}$, nebulizer to $70 \mathrm{psi}$. Maximal accumulation time was fixed to $300 \mathrm{~ms}$ and the loading of the trap was controlled by the instrument with an ICC of 30,000

\subsection{Surface analysis}

\subsubsection{Contact angle measurement}

Contact angles of the PTT polymeric films before and after enzymatic treatment were measured with the Drop Shape Analysis System DSA 100 (Krüss GmbH, Hamburg, Germany). Deionized water was used as test liquid with a drop size of $3 \mu \mathrm{l}$. Contact angle was measured after $3 \mathrm{~s}$ and data are obtained from the averages of the measurements taken from 10 different points of the sample surface.

\subsubsection{Environmental scanning electron microscopy (ESEM)}

ESEM was used to analyze surface morphology of white PTT fabric after either treatment with cutinase from T. fusca or alkaline treatment. The equipment used for analysis was an ESEM Quanta 600F with a Schottky emitter (FEI, Eindhoven, Netherlands). Measurements were carried out in low vacuum at $93 \mathrm{~Pa}$ using water vapour as gaseous environment. Applied accelerating voltage was $10 \mathrm{kV}$ by means of a large field detector.

\subsection{Dyeing and colour measurements}

For dyeing experiments the fabrics were washed thoroughly, i.e. twice with $\mathrm{Na}_{2} \mathrm{CO}_{3}\left(2 \mathrm{gl}^{-1}\right)$ for $30 \mathrm{~min}$, twice with deionised water (30 min) and additionally soxhlet extracted overnight with denatured ethanol to remove attached protein. Dyeing was carried out with basic dye Astrazon Blue BG (C.I. Basic Blue 3). A dyestuff solution of $0.05 \%(\mathrm{w} / \mathrm{v})$ with deionised water was prepared and enzymatically treated PTT white fabrics were dyed for $10 \mathrm{~min}$ at room temperature on a rotary shaker at $40 \mathrm{rpm}$. Colour measurements were carried out on a Spectraflash ST 600 Plus (Datacolor International) with a wavelength range of $380-700 \mathrm{~nm}$. For characterisation of the dyed samples reflectance measurements were carried out and K/S values compared at the wavelength of $660 \mathrm{~nm}$ (Denter et al., 1987).

\section{Results and discussion}

The potential of enzymes for surface hydrolysis of poly(trimethylene terephthalate) (PTT) was assessed in this study. Since this is the first such attempt there is no information on PTT hydrolysing enzymes in the literature. However, in the last few years surface hydrolysis of poly(ethylene terephthalate) (PET) has been reported by various groups including our lab (Liebminger et al., 2007; Heumann et al., 2006; Alisch et al., 2004; Vertommen et al., 2005; Nimchua et al., 2007). Thus, we have used enzymes with known activity on PET from $T$. lanuginosus, P. citrinum, F. solani and T. fusca in this study.

\subsection{Bis(3-hydroxypropyl) terephthalate (BHPT) as model substrate}

An essential element in the study of biotransformation of PTT was the design and synthesis of a model substrate for this polymer.

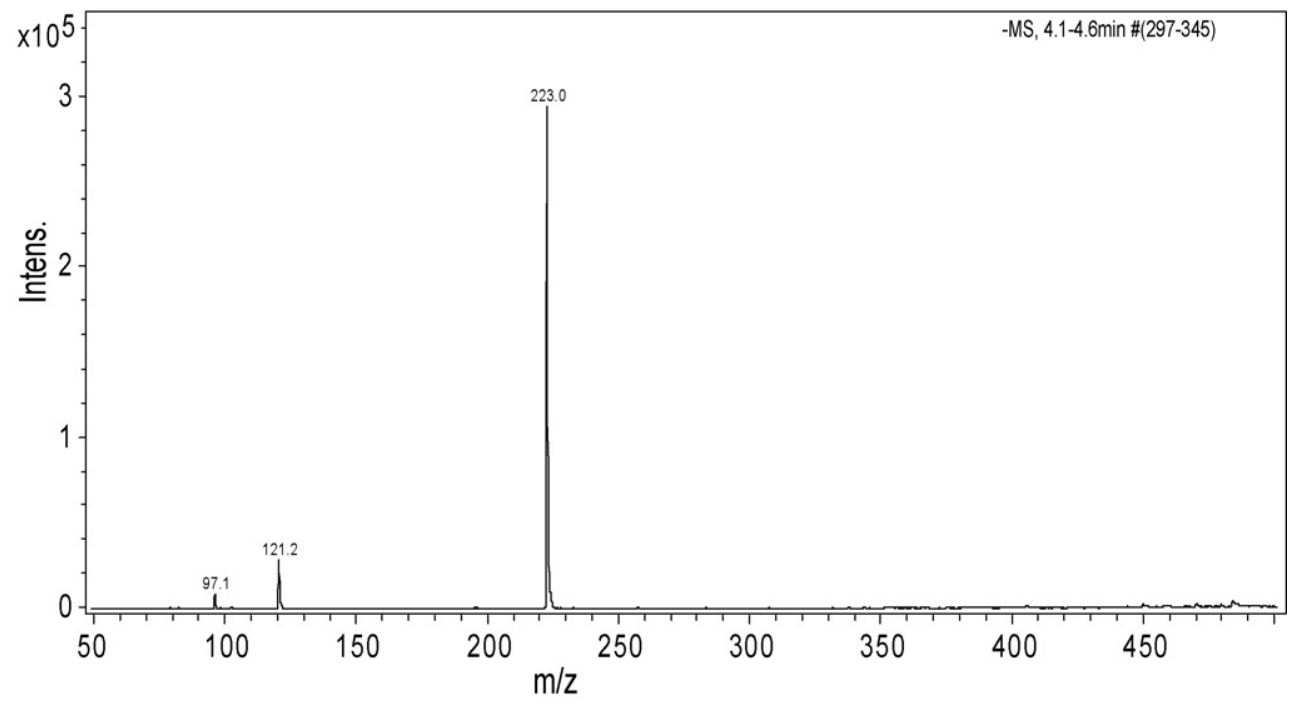

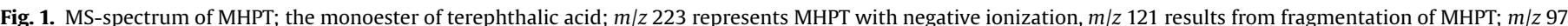
is ubiquitous and most probably resulting from plasticizers. 
As obvious from the results below, enzymatic hydrolysis of PTT is a slow process and changes on the polymer surface are difficult to quantify. In contrast, hydrolysis of a short chain model substrate should be faster and analysis of transformation products easier. This is essential not only for mechanistic studies but also for the future development of efficient screening procedures for PTT-hydrolases.

The synthesis of BHPT substrate was done in one step with a yield of $63.1 \%$. The structure was confirmed with NMR and MS. For the potential hydrolysis product of BHPT, the propyleneglycol monoester of terephthalic acid (MHPT) no reference substance is commercially available. Thus, identification and confirmation of MHPT was carried out by HPLC/MS (Fig. 1) using MS-compatible buffers and acids. Ionization was achieved with electrospray in negative mode.

To study biotransformation of PTT, four enzymes which have previously been described to hydrolyse PET have been compared. Interestingly, these "polyesterases" cannot be classified to one distinct EC class of enzymes and PET-hydrolase activity was previously found for both lipases ( $T$. lanuginosus, ) proteases (P. citrinum) and cutinases (F. solani pisi, T. fusca) (Heumann et al., 2006; Liebminger et al., 2007; Nimchua et al., 2007). For this reason, it is impossible to compare the specificities of these enzymes on polyesters based on an identical "standard" activity dosed (e.g. p-nitro-phenol substituted fatty acids).

Out of the tested enzymes the lipase from T. lanuginosus and the cutinase from $T$. fusca were able to hydrolyse the PTT model substrate while the other enzymes did not show significant activity. For this reason the T. fusca and T. lanuginosus enzymes were chosen as the representatives of cutinases and lipases for this study, respectively. To compare the substrate specificities, the $T$. fusca cutinase and the T. lanuginosus lipase were used in protein concentrations leading to a clearly measurable turnover of BHPT after the same incubation time.

During incubation of BHPT in aqueous solution with the cutinase from T. fusca and the lipase from T. lanuginosus, the amount of BHPT decreased gradually with time. Some autohydrolysis of BHPT in buffer solution at both incubation temperatures was accounted for in all calculations.

After initial attack of the ester bond of a BHPT molecule by the $T$. fusca cutinase, the enzyme did not further hydrolyse the ester bond of the resulting MHPT molecule. Instead it continued hydrolysis of BHPT. Only after complete conversion of BHPT to MHPT, the T. fusca cutinase started to hydrolyse the remaining ester bonds from MHPT to form terephthalic acid (TA) (Fig. 2).

Interestingly, in contrast to these results achieved with the $T$. fusca cutinase, no TA could be detected after treatment with the $T$. lanuginosus lipase, - even after $24 \mathrm{~h}$ of incubation (Fig. 3 ). Thus, the

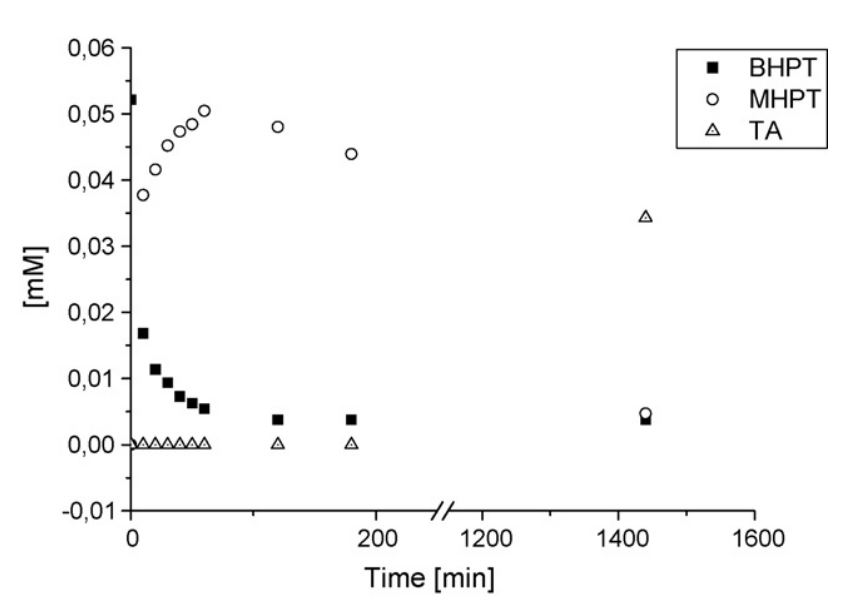

Fig. 2. Hydrolysis of BHPT with cutinase from T. fusca.

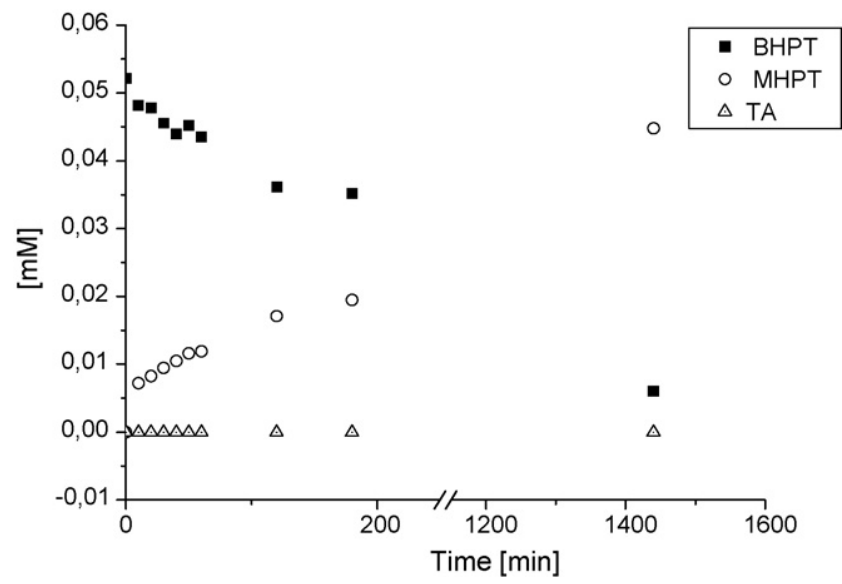

Fig. 3. Hydrolysis of BHPT with lipase from T. lanuginosus.

lipase seems to be unable to hydrolyse MHPT. This already indicates different modes of action of the enzymes. It is known that lipases often do not have the ability to cleave dissolved substances whereas the $T$. fusca cutinase displays significant activity to this class of substrates (Mueller et al., 2005).

\subsection{Hydrolysis of cyclic PTT oligomers}

Cyclic dimers of PTT are unwanted by-products during PTTproduction. Enzymatic hydrolysis of cyclic oligomers of aromatic polyesters was already shown for PET, where the main by-product during polycondensation to form PET consists of a cyclic trimer (Hooker et al., 2002). The crucial step in the enzymatic hydrolysis of the cyclic species is the cleavage of the cyclic structure while thereafter hydrolysis will continue fast until almost all ester bonds are hydrolysed. In this study, the T. lanuginosus lipase was not able to open the cyclic PTT structure and no hydrolysis products were detected.

With the cutinase from T. fusca, however, activity on the cyclic dimer of PTT was clearly detected (Figs. 4 and 5). No BHPT was detected upon incubation of the cyclic dimer with the cutinase from T. fusca which is in line with the results of the hydrolysis experiments of BHPT. Thus, BHPT resulting during sequential hydrolysis of the cyclic dimer was quickly hydrolysed to give MHPT. However, the low content of TA at the beginning of the reaction rather indicates that BHPT is never formed and that the opened dimer is

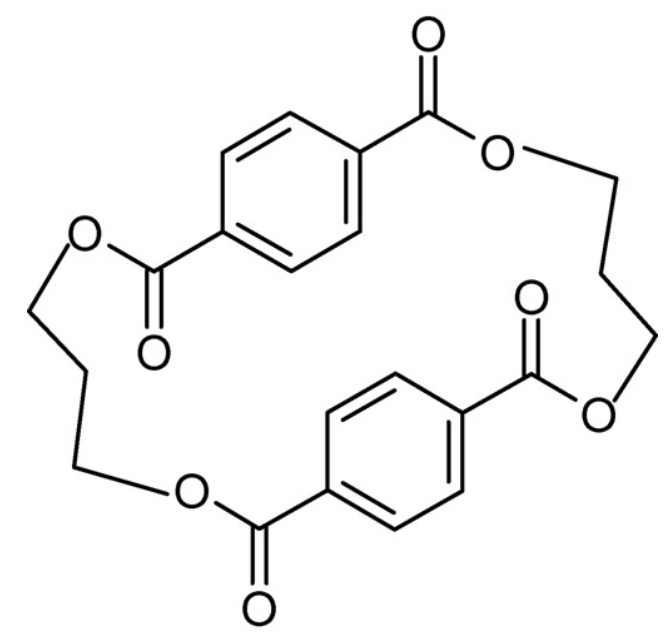

Fig. 4. Structure of the cyclic dimer of PTT. 


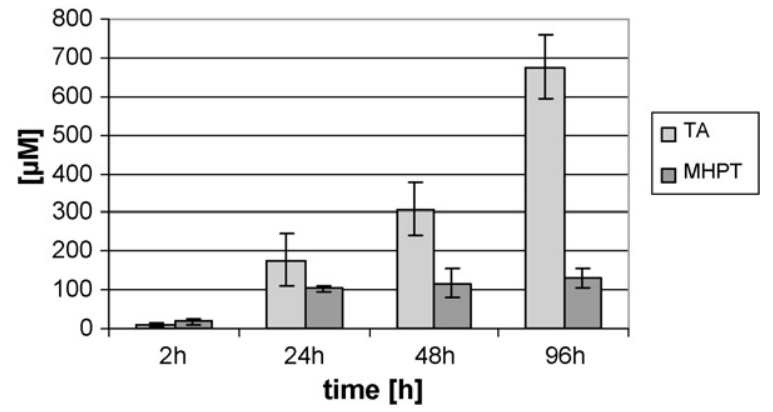

Fig. 5. Hydrolysis of the cyclic dimer of PTT with a cutinase from T. fusca.

hydrolysed in the middle to give two molecules of MHPT. The ratio of MHPT to TA decreases over incubation time, which again indicates the formation of TA by cleaving the remaining ester bond in MHPT after ring opening.

Previously, it has been reported that the ratio of the ethyleneglycol monoester of TA to TA detected in solution also depends on the enzyme to substrate ratio. This research was carried out with the cutinase from $F$. solani pisi for poly(ethylene terephthalate) and its cyclic trimer, respectively (Vertommen et al., 2005; Hooker et al., 2002).

\subsection{Hydrolysis of PTT polymers}

A major parameter changing upon surface hydrolysis of aromatic polyesters is the hydrophilicity (Alisch et al., 2004; Alisch-Mark et al., 2006; Fischer-Colbrie et al., 2004). However, apart from the enzymatic introduction of polar hydroxyl/carboxyl groups onto the polymer surface, protein from the enzyme solution potentially adsorbing to the polymer might also contribute to the hydrophilicity increase (Vertommen et al., 2005). Therefore, hydrolysis experiments of PTT films were carried out with the additional study of the influence of $\mathrm{HgCl}_{2}$ as inhibitor (Fig. 6). In the case of PTT-films, only the cutinase from T. fusca was able to hydrolyse the polymer. An increase of the hydrolysis products terephthalic acid (TA) and mono(3-hydroxypropyl terephthalate) MHPT was observed for up to $120 \mathrm{~h}$ of incubation at $60^{\circ} \mathrm{C}$. Again, as already observed in the case of the cyclic dimer, no BHPT was detected while the ratio of MHPT to TA also decreased. This would again indicate that terminal TA is not preferentially released from polymers/oligomers. Upon inhibition with $\mathrm{HgCl}_{2}$, significant lower amounts of TA and MHPT were detectable although inhibition was not complete.

The increase of hydrophilicity after the enzymatic treatment of the films was verified via contact angle measurements (Lai et al., 2006). Recent research in surface analysis after enzymatically treated PET-films showed that enzymes bind very strongly to the polymer surfaces (Vertommen et al., 2005). Even after several washing procedures of the treated films it was shown by ESCA

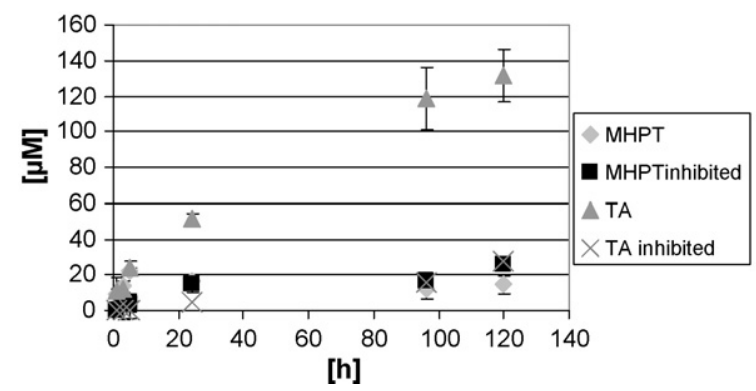

Fig. 6. Hydrolysis of films with cutinase from $T$. fusca and inhibition with $\mathrm{HgCl}_{2}$.

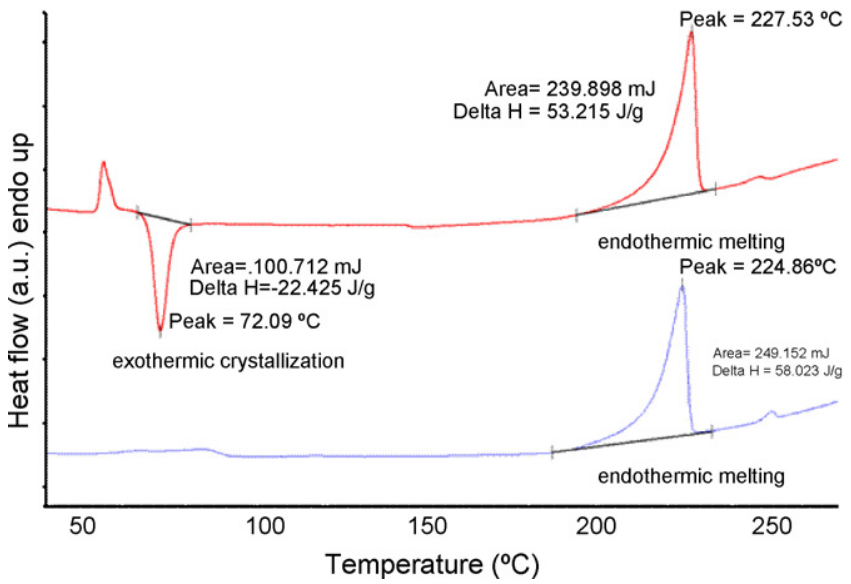

Fig. 7. DSC diagram of the PTT sample before treatment (upper line) and after blank incubation for $1 \mathrm{~h}$ (lower line).

measurements that no complete removal of the proteins from the surface was possible. Therefore results obtained by HPLC cannot be correlated to surface modification of the fibres since an increase in wettability cannot only be referred to the action of the enzymes alone but also to their adsorption to the surface.

Pieces of PTT films were incubated in the size of $4 \mathrm{~cm} \times 4 \mathrm{~cm}$ with the cutinase from $T$. fusca to have enough flat surface area for reliable contact angle data. Treating the PTT films with inhibited enzymes the contact angles of these articles should be significantly higher than with the native enzymes to assure their modification ability. Contact angles of the films after treatment with the native enzyme were $94.4 \pm 2.6^{\circ}$ and with the inhibited enzyme $96.1 \pm 3.9^{\circ}$ compared to the reference film with $105.3 \pm 4.26^{\circ}$. Since contact angles after treatment with the inhibited enzyme are also lower than the reference it can be assumed that there is still protein adsorbed to the surface as already shown by Vertommen et al. (2005).

Previously it has been reported for PET that polyesterases preferentially attack the amorphous regions of the polymer. Consequently, the degree of crystallinity of the polymer should increase upon enzyme treatment (Vertommen et al., 2005; Herzog et al., 2006; Mueller, 2006; Mueller et al., 2005). The PTT film investigated in this work showed a significant crystallisation peak in DSC analysis before incubation. The degree of crystallization was calculated from the difference in enthalpies of the melting peak and the crystallization peak. With the assumption of $145 \mathrm{Jg}^{-1}$ melting enthalpy for a $100 \%$ crystalline PTT a degree of crystallinity of $21 \%$ was assumed.

The cutinase from $T$. fusca has its activity optimum at $65^{\circ} \mathrm{C}$ and is known to be thermostable for a period of time at $55^{\circ} \mathrm{C}$ (Mueller,

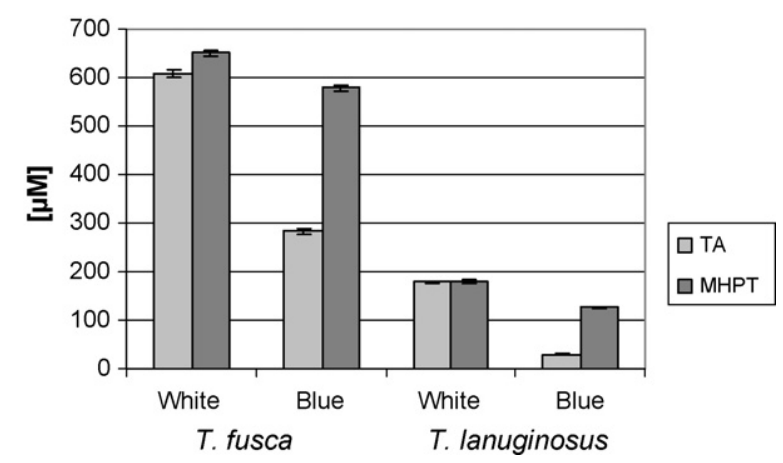

Fig. 8. Hydrolysis of PTT-fabrics after $72 \mathrm{~h}$ incubation with $T$. fusca and T. lanuginosus enzymes. 


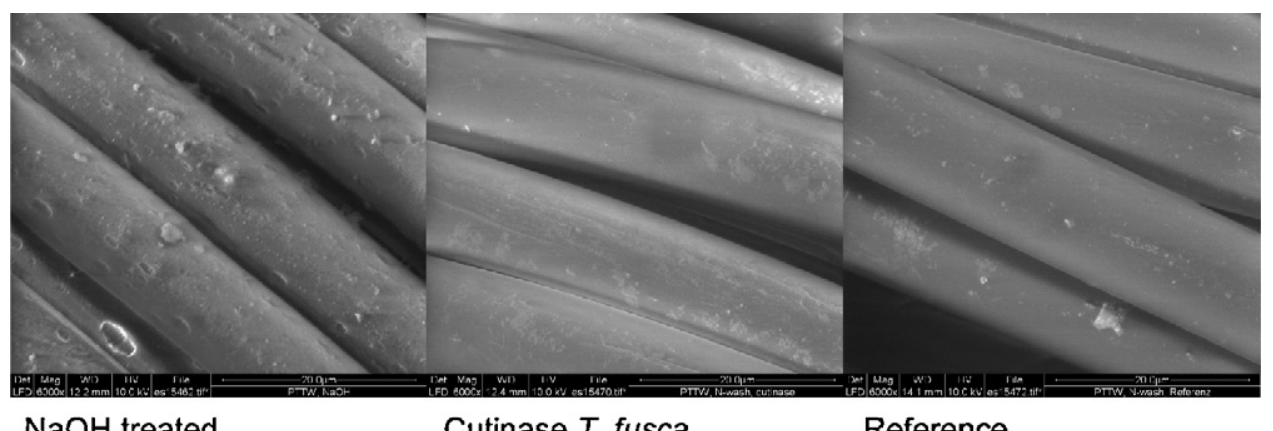

$\mathrm{NaOH}$ treated

Cutinase T. fusca

Reference

Fig. 9. ESEM images after treatment with sodium hydroxide and cutinase from $T$. fusca compared to a reference.

2006). The experiments in this work were carried out at $60^{\circ} \mathrm{C}$ to ensure high chain mobility in the PTT chains. This is achieved, when the glass transition temperature $\left(T_{\mathrm{g}}\right)$ is close to the temperature at which hydrolysis is carried out (Welzel, 2003). Buffer incubations for blank measurements were carried out as well. When subjecting the polymer to DSC just after $1 \mathrm{~h}$ shaking at $60^{\circ} \mathrm{C}$ the crystallization peak completely disappeared (Fig. 7). Since the incubation temperature was higher than the glass transition temperature, which is in the range of $42-72{ }^{\circ} \mathrm{C}$ (Huang and Chang, 2000; Pyda et al., 1998 ) the polymer was annealing during the treatment resulting in higher crystallinity of about $38 \%$. But obviously still enough amorphous areas and crystal domains with sufficient chain flexibility were available for the enzymes since hydrolysis products were still increasing up to 5 days (Fig. 6).

Repeating the experiments at a temperature of $40^{\circ} \mathrm{C}$ the polymer did not show any hydrolysis products. This working temperature was too low to obtain reasonable hydrolysis of the films with the cutinase from T. fusca. Obviously the activity of enzymes on polymers is not necessarily dependent on high incubation temperatures since Vertommen et al. (2005) could degrade amorphous poly(ethylene terephthalate) films with a cutinase from $F$. solani pisi at already $30^{\circ} \mathrm{C}$, although the chain mobility of the polymer correlates with the difference between the melting point of the polyester and the hydrolysis temperature (Marten et al., 2005).

In a next stage, two different PTT fabrics which both did not show a crystallization peak were treated with the cutinase from $T$. fusca. The calculations gave the degree of crystallinity of $45 \%$ for the dyed (blue) and $37 \%$ for the undyed (white) fabric. Interestingly, in the case of the fabrics, the lipase displayed hydrolytic activity, which was the case neither with the PTT film nor with the cyclic dimer. Compared to the PTT film higher amounts of TA and MHPT were detected. This is definitely due to the larger surface area of the fabrics compared to the films, since the fabrics consist of single threads. The higher amounts of hydrolysis products in the case of the undyed fabric compared to the dyed one can probably be referred to the lower crystallinity of the undyed white fabric (Fig. 8). The effective surface area of the fabrics was not determined and therefore this might be another point contributing to the higher amount of hydrolysis products. Possible changes in hydrophilicity at the surface could not be demonstrated, since neither water dissipation experiments nor contact angle measurements could be carried out on the fabrics due to the irregular morphology of the surface.

Morphology changes and dyeability of PTT fabrics were assessed with undyed white fabric only. The cutinase from T. fusca was chosen for the pre-treatment due to its higher cleavage ability on PTT. ESEM images from alkaline treated fabrics were compared to enzymatically treated ones. As can be impressively seen from Fig. 9, alkaline hydrolysis results in crater like structures on the fibres indicating drastic degradation of the fabric. T. fusca treated fabrics did not show any difference in surface morphology compared to the reference, which was treated in buffer solution. Therefore it can be concluded that the surface structure is not damaged and mechanical stability is still maintained after treatment with enzymes. After alkaline treatment "pitting corrosion" is visible subsequently leading to a weakening of the fabric. Weight loss results could affirm this again, since after just half an hour of alkaline treatment at $95^{\circ} \mathrm{C}$ a loss of about $16 \%$ was measured. Compared to cutinase exposed fabric this is very high, since for this enzyme even after four days of incubation at $60^{\circ} \mathrm{C}$ just a loss of around $1 \%$ could be verified.

Dyeing experiments were carried out for undyed PTT fabric after incubation with cutinase from T. fusca. Treatment with the enzyme and careful washing resulted in a significant increase of $\mathrm{K} / \mathrm{S}$ values from 0.21 to 0.74 . Astrazon Blue BG is a cationic dye and hence reacting with anionic carboxylic groups. This once more indicates the endo-chain-cleavage properties of the T. fusca enzyme.

\section{Conclusion}

In this work it was clearly demonstrated that cutinase from T. fusca was able to hydrolyse the aromatic polyester poly(trimethylene terephthalate) based on the detection of the water soluble hydrolysis products TA and MHPT with HPLC-UVD. The T. fusca enzyme hydrolysed both PTT fibres and films whereas the lipase from T. lanuginosus was only able to hydrolyse the fibres. The already discussed influence of crystallinity of the polymers plays an important part in the studies of modification of synthetic polymers. Due to the higher surface area obviously fibres are easily attacked by enzymes than films. Cyclic PTT dimers were only opened and hydrolysed by the T. fusca cutinase. In dyeing, a clear increase of $\mathrm{K} / \mathrm{S}$ values after cutinase treatment could be measured without structural damages of the fibre compared to alkaline treatment. Thus, in PTT production and processing this enzyme has a high potential for PTT surface modification and removal of cyclic dimers.

\section{Acknowledgements}

Many thanks to Markus List for synthesis of BHPT, to Matthias Pretzler and Ulrike Geweßler for lab work, to Carina Illaszewicz and Hansjoerg Weber for providing NMR spectra and to Gregor Trimml for DSC measurements. Further thanks to R.J. Mueller from GBF, Braunschweig, Germany and to Shell Chemicals for providing enzymes and PTT knit fabrics, respectively, and to Zahra Ahmadi for making PTT films. The research was financed by the SFG, the FFG, the city of Graz and the province of Styria.

\section{References}

Alisch, M., Feuerhack, A., Mueller, R.J., Mensak, B., Andreaus, J., Zimmermann, W., 2004. Biocatalytic modification of polyethylene terephthalate 
fibres by esterases from actinomycete isolates. Biocatal. Biotransform. 22, 347-351.

Alisch-Mark, M., Herrmann, A., Zimmermann, W., 2006. Increase of the hydrophilicity of polyethylene terephthalate fibres by hydrolases from Thermomonospora fusca and Fusarium solani f. sp. pisi. Biotechnol. Lett. 28, 681-685.

Araujo, R., Silva, C., O’Neill, A., Micaelo, N., Guebitz, G., Soares, C.M., Casal, M., CavacoPaulo, A., 2007. Tailoring cutinase activity towards polyethylene terephthalate and polyamide 6,6 fibers. J. Biotechnol. 128, 849-857.

Chen, X., Hou, G., Chen, Y., Yang, K., Dong, Y., Zhou, H., 2007. Effect of molecular weight on crystallization, melting behavior and morphology of poly(trimethylene terephthalate). Polym. Testing 26, 144-153.

Denter, U., Elgert, K.-E., Heidemann, G., 1987. Farbtest zur Charakterisierung abgeschälter Polyesteroberflächen. Melliand Textilber 11, 848.

Dresler, K., Van den Heuvel, J., Mueller, R.-J., Deckwer, W.-D., 2006. Production of a recombinant polyester-cleaving hydrolase from Thermobifida fusca in Escherichia coli. Bioproc. Biosyst. Eng. 29, 169-183.

Fischer-Colbrie, G., Heumann, S., Liebminger, S., Almansa, E., Cavaco-Paulo, A. Guebitz, G.M., 2004. New enzymes with potential for PET surface modification. Biocatal. Biotransform. 22, 341-346.

Gouda, M.K., Kleeberg, I., Van den Heuvel, J., Mueller, R.J., Deckwer, W.D., 2002. Production of a polyester degrading extracellular hydrolase from Thermomonospora fusca. Biotechnol. Progr. 18, 927-934.

Herzog, K., Mueller, R.J., Deckwer, W.D., 2006. Mechanism and kinetics of the enzymatic hydrolysis of polyester nanoparticles by lipases. Polym. Degrad. Stab. 91, 2486-2498.

Heumann, S., Eberl, A., Pobeheim, H., Liebminger, S., Fischer-Colbrie, G., Almansa E., Cavaco-Paulo, A., Gubitz, G.M., 2006. New model substrates for enzymes hydrolysing polyethyleneterephthalate and polyamide fibres. J. Biochem. Biophys. Meth. 69, 89-99.

Hooker, J., Hinks, G., Montero, G., Icherenska, M., 2002. Enzyme-catalyzed hydrolysis of poly(ethylene terephthalate) cyclic trimer. J. Appl. Polym. Sci. 89, 2545-2552.

Houck, M.M., Huff, R.A., Lowe, P.C., Menold, R.E., 2001. Poly(trimethylene terephthalate): a "new" type of polyester fiber. Forensic Sci. Commun., 3.

Huang, J.M., Chang, F.C., 2000. Crystallization kinetics of poly(trimethylene terephthalate). J. Polym. Sci.: Part B: Polym. Phys. 38, 934-941.

Kleeberg, I., Welzel, K., VandenHeuvel, J., Mueller, R.J., Deckwer, W.D., 2005 Characterization of a new extracellular hydrolase from Thermobifida fusca degrading aliphatic-aromatic copolyesters. Biomacromolecule 6, 262270.

Lai, J., Sunderland, B., Xue, J., Yan, S., Zhao, W., Folkard, M., Michael, B.D., Wang, Y., 2006. Study on hydrophilicity of polymer surfaces improved by plasma treatment. Appl. Surf. Sci. 252, 3375-3379.
Liebminger, S., Eberl, A., Fischer-Colbrie, G., Heumann, S., Guebitz, G.M., 2007. Hydrolysis of PET and bis (benzoyloxyethyl) terephthalate with a new polyesterase from Penicillium citrinum. Biocat. Biotrans. 25, 171-177.

Lowry, O.H., Rosebrough, N.J., Farr, A.L., Randall, R.J., 1951. Protein measurement with the Folin phenol reagent. J. Biol. Chem. 193, 265-275.

Lyoo, W.S., Geun Lee, S., Shik Ha, W., Lee, J., Ho Kim, J., 2000. A high performance liquid chromatography method to determine monomer reactivity ratios in copolycondensation of bis(4-hydroxybutyl) terephthalate and bis(2-hydroxyethyl) terephthalate. Polym. Testing 19, 299-309.

Marten, E., Mueller, R.J., Deckwer, W.-D., 2005. Studies on the enzymatic hydrolysis of polyesters. II. Aliphatic-aromatic copolyesters. Polym. Degrad. Stab. 88, 371-381.

Marten, E., Mueller, R.J., Deckwer, W.D., 2003. Studies on the enzymatic hydrolysis of polyesters I. Low molecular mass model esters and aliphatic polyesters. Polym. Degrad. Stab. 80, 485-501.

Mueller, R.J., 2006. Biological degradation of synthetic polyesters-enzymes as potential catalysts for polyester recycling. Proc. Biochem. 41, 2124-2128.

Mueller, R.J., Kleeberg, I., Deckwer, W.D., 2001. Biodegradation of polyesters containing aromatic constituents. J. Biotechnol. 86, 87-95.

Mueller, R.J., Schrader, H., Profe, J., Dresler, K., Deckwer, W.D., 2005. Enzymatic degradation of poly(ethylene terephthalate): rapid hydrolyse using a hydrolase from T. fusca. Macromol. Rapid Commun. 26, 1400-1405.

Nimchua, T., Punnapayak, H., Zimmermann, W., 2007. Comparison of the hydrolysis of polyethylene terephthalate fibers by a hydrolase from Fusarium oxysporum LCH I and Fusarium solani f. sp. pisi. Biotechnol. J. 2, 361-364.

Pang, K., Kotek, R., Tonelli, A., 2006. Ring-opening polymerization of the cyclic dimer of poly(trimethylene terephthalate). J. Polym. Sci.: Part A: Polym. Chem. 44, 6801-6809.

Pyda, M., Boller, A., Grebowicz, J., Chuah, H., Lebedev, V., Wunderlich, B., 1998. Heat capacity of poly(trimethylene terephthalate). J. Polym. Sci.: Part B: Polym. Phys. $36,2499-2511$.

Sichina, W.J., 2000. DSC as problem solving tool: measurement of percent crystallinity of thermoplastics. Thermal Analysis Application Note.

Silva, C., Carneiro, F., OǐNeill, A., Fonseca, L.P., Cabral, J.M.S., Guebitz, G., Cavaco-Paulo, A., 2005. Cutinase-a new tool for biomodification of synthetic fibers. J. Polym. Sci. 43, 2448-2450.

Vertommen, M.A.M.E., Nierstrasz, V.A., Veer, M.v.d., Warmoeskerken, M.M.C.G., 2005. Enzymatic surface modification of poly(ethylene terephthalate). J. Biotechnol. 120, 376-386.

Welzel, K., 2003. Einfluss der chemischen Struktur auf die enzymatische Hydrolyse von Polyester-Nanopartikeln. Dissertation TU Braunschweig, Germany.

Wu, T., Li, Y., Wu, Q., Song, L., Wu, G., 2005. Thermal analysis of the melting process of poly(trimethylene terephthalate) using FTIR micro-spectroscopy. Eur. Polym. J. 41, 2216-2223 